Interactive comment on "ESMValTool v2.0 Extended set of large-scale diagnostics for quasi-operational and comprehensive evaluation of Earth system models in CMIP" by Veronika Eyring et al.

Veronika Eyring et al.

veronika.eyring@dlr.de

Received and published: 19 March 2020

\title{
Reply to Martine Michou
}

We thank Martine Michou for the helpful comments. We have now revised our manuscript in light of these and the other review comments we have received. A pointwise reply is given below.

The text mentions that Figures 42 and 43 consist of an adaptation of di- 
agnostics included in the CCMValDiag tool. It is very valuable to have recipe_eyring06jgr.yml in v2.0 of ESMValTool (it is not yet the case as of January 2020), as well as a recipe with all diagnostics of Eyring et al, JGR 2013, "Longterm ozone changes and associated climate impacts in CMIP5 simulations" that includes interesting diagnostics with regards to ozone evaluation.

Thanks for your interest in the inclusion of the diagnostics from the Eyring et al., JGR, 2006 and 2013 papers on ozone evaluation. We agree that it would be good to have them available in ESMValTool v2. A subset of the diagnostics from Eyring et al., JGR, 2006 (e.g. Figures 42 and 43) are part of the official release of version 2 of the ESMValTool. These are described in the manuscript. Additional diagnostics from these two papers will be added as ressources allow.

Second, as of January 2020, it is not easy at all to install ESMValTool v2.0, unlike v1.0. For users without conda, that is only recommended in the install web page, the installation with the "pip install ." command, as written in the install web page does not work. Documentation should be amended, in particular for the use of more "dummy" users, if ESMValTool v2.0 is to be used by a wide community. And I think it deserves it.

Installing the ESMValTool without using conda is not possible at the moment. The installation procedure is clearly described in the ESMValTool documentation on ReadTheDocs (https://esmvaltool.readthedocs.io/en/latest/getting_started/install.html) and has been successfully applied by all users and developers so far. Specific installation issues (which might arise, for example, on some peculiar architectures) can be reported on the GitHub page of the ESMValTool and are readily addressed by the development team.

Printer-friendly version

Discussion paper 
Interactive comment on Geosci. Model Dev. Discuss., https://doi.org/10.5194/gmd-2019-291, 2019.

Interactive

comment 